\title{
COMPACTNESS PROPERTIES OF TOPOLOGICAL GROUPS. III
}

\author{
BY
}

\author{
S. P. WANG ${ }^{1}$ )
}

\begin{abstract}
Compactness properties of topological groups and finiteness of Haar measure on homogeneous spaces are studied. Some concrete structure theorems are presented.
\end{abstract}

Finiteness properties of groups have been studied by R. Baer [1] and B. H. Neumann [9]. For topological groups, the analogous compactness properties were studied by V. I. Ušakov [13] , [14], [15] , J. Tits [12] and the author [17], [18] . The present work is a continuation of [17], [18]. Some concrete structure theorems of topological groups are established.

Let $G$ be a topological group. An element $x$ of $G$ is called periodic if $x$ is contained in a compact subgroup of $G$. We denote by $P(G)$ the set of all periodic elements of $G$. An element $x$ of $G$ is called conjugate-bounded, or simply bounded, if the conjugacy class $\left\{\mathrm{gxg}^{-1}: g \in G\right\}$ is relatively compact in $G$. We write $F(G)$ for the set of all conjugate-bounded elements of $G$. In this paper, we discuss compactness properties related to $F(G)$ and $P(G)$.

Let $G$ be a locally compact group. If $G$ is connected, $G$ has a unique maximal compact normal subgroup. In general, this is not true. In the first section, we define a radical $W(G)$ of $P(G)$ such that $W(G)$ is a closed characteristic subgroup of $G$ contained in $P(G)$ which has analogous properties of maximal compact normal subgroups. We have proved the following theorems.

THEOREM 1.5. Let $G$ be a locally compact group and $W(G)$ the radical of $P(G)$. Then $W(G)$ has the following conditions:

(i) $W(G)$ is a closed characteristic subgroup of $G$.

(ii) Every relatively compact subset $X$ of $W(G)$ generates a relatively compact subgroup $\langle X\rangle$ of $G$; in particular $W(G) \subset P(G)$.

(iii) The inverse image of $P(G / W(G))$ in $G$ is $P(G)$.

(iv) $G / W(G)$ has no nontrivial compact normal subgroups; moreover $W(G)$ is characterized to be the smallest closed normal subgroup $N$ of $G$ such that $G / N$ has no nontrivial compact normal subgroups.

\footnotetext{
Received by the editors June 27, 1974 .

AMS (MOS) subject classifications (1970). Primary 22D05; Secondary 22E15.

Key words and phrases. Locally compact groups, Lie groups, periodic elements, bounded elements, Haar measure.

( $\left.{ }^{1}\right)$ Partially supported by N. S. F. Grant GP-37688. 
(v) If $G$ has a maximal compact normal subgroup $K$, then $W(G)=K$.

THEOREM 1.6. If $G$ is a finitely generated discrete group, then $W(G)=$ $F(G) \cap P(G)$.

In $\S 2$, we consider closed subgroups of $G$ which are contained in $F(G)$. Here we present the following theorems.

THEOREM 2.3. Let $G$ be a locally compact group and $H$ a subgroup of $F(G)$. Then the following conditions are equivalent:

(i) $\mathrm{Cl}(H) \subset F(G)$.

(ii) For every subset $X$ of $H$ relatively compact in $G$, the union of $g \mathrm{Xg}^{-1}$ $(g \in G)$ is relatively compact in $G$.

THEOREM 2.4. Let $G$ be a locally compact group. Then the following conditions are equivalent:

(i) $F(G) \cap P(G)$ is closed in $G$.

(ii) Every subset $X$ of $F(G) \cap P(G)$ relatively compact in $G$ is contained in a compact normal subgroup of $G$.

In $\S 3$, we discuss closed normal subgroups $H$ of $G$ with $\mathrm{Cl}(H \cap F(G))=H$. We prove the following structure theorem.

THEOREM 3.8. Let $G$ be a locally compact group, $H$ a closed normal subgroup of $G$ with $H=\mathrm{Cl}(H \cap F(G))$. Then we have the following conditions:

(i) $P(H)=\mathrm{Cl}(H \cap F(G) \cap P(G))$.

(ii) $H^{0} \subset F(G)$.

(iii) $H=(H \cap F(G)) P(H)$.

(iv) $H / P(H)$ is the direct product $V \times D$ where $V$ is a normal vector subgroup of $G / P(H)$, and $D$ a normal discrete torsion free abelian subgroup of $G / P(H)$ with $D \subset F(G / P(M))$.

(v) $H / K$ is the direct product $W \times T$ where $K$ is the maximal compact normal subgroup of $H^{0}, W$ a normal vector subgroup of $G / K$ and $T$ a totally disconnected normal subgroup of $G / K$ with $T=\mathrm{Cl}(T \cap F(G / K))$.

Applying Theorem 3.8 to compactly generated normal subgroup $H$, we have a structure theorem, Theorem 3.10, for elements in $F(G)$. We also generalize the results of Tits in [12].

In $\S \S 4$ and 5, we discuss the relationship between compactness and finiteness of Haar measure. We have the following theorems.

THEOREM 4.5. Let $G$ be a Lie group and $H$ a closed subgroup of $G$ such that the Lie algebra $\hat{G}$ of $G$ has no noncompact semisimple subalgebras. Then the following two conditions are equivalent: 
(i) $G / H$ is compact.

(ii) $G / H$ has a finite invariant Haar measure.

THEOREM 5.6. Let $G$ be a locally compact group and $\Lambda$ a subset of the automorphism group $A(G)$ of $G$ and $Z_{G}(\Lambda)=\{g \in G: \lambda(g)=g \forall \lambda \in \Lambda\}$. If $G / Z_{G}(\Lambda)$ has a finite invariant Haar measure, then $G$ has an open subgroup $H$ such that $H /\left(H \cap Z_{G}(\Lambda)\right)$ is compact.

Finally we prove that under some mild assumption on $G$ that for an element $g \in F(G), G / Z_{G}(g)$ is compact if and only if $G / Z_{G}(g)$ has finite invariant volume.

Notations. Let $G$ be a topological group. The identity element of $G$ is denoted by $e$ and the identity component of $G$ is given by $G^{0}$. Given subsets $X$, $Y$ of $G$, we write $C(X, Y)$ for the subset $\left\{x y x^{-1}: x \in X, y \in Y\right\}$. Let $X$ be a subset of $G$. $\mathrm{Cl}(X)$ is the closure of $X$ in $G$. By an analytic group, we mean a connected Lie group.

1. The radical of $P(G)$. Let $G$ be a locally compact group and $P(G)$ the subset of all periodic elements of $G$. If $G$ is connected, it is well known that $G$ has a unique maximal compact normal subgroup. However the existence of maximal compact normal subgroup is not true in general. In this section, we are going to define a radical $W(G)$ of $P(G)$. $W(G)$ is a closed characteristic subgroup of $G$ contained in $P(G)$ which has analogous properties of a maximal compact normal subgroup. In case $G$ has a maximal compact normal subgroup $K, W(G)=K$. We define inductively $W_{1}(G)=\mathrm{Cl}(P(G) \cap F(G)), W_{\lambda+1}(G)$ the preimage of $W_{1}\left(G / W_{\lambda}(G)\right)$ in $G$, and if $\lambda$ is a limiting ordinal number $W_{\lambda}(G)=\mathrm{Cl}\left(\bigcup_{\nu<\lambda} W_{\nu}(G)\right)$.

Definition 1.1. Let $G$ be a locally compact group. The radical $W(G)$ of $P(G)$ is the group $W_{\beta}(G)$ with $W_{\beta}(G)=W_{\beta+1}(G)$.

Before we present the properties of the groups $W_{\lambda}(G)$ and $W(G)$, we shall first establish a lemma.

LEMma 1.2. Let $G$ be a locally compact group, $H$ a closed normal subgroup of $G$, and $\pi: G \rightarrow G / H$ the projection map. Suppose that $H$ has the property that every relatively compact subset $X$ of $H$ generates a relatively compact subgroup $\langle X\rangle$ of $H$. If $Y$ is a relatively compact subset of $G$ such that the group $\pi(\langle Y\rangle)$ is relatively compact in $G / H$, then the group $\langle Y\rangle$ is relatively compact in $G$.

Proof. Since $\pi(\langle Y\rangle)$ is relatively compact, there is a compactly generated closed subgroup $M$ of $G$ containing $Y$ such that $\pi(M)$ is compact. Since $M$ is $\sigma$-compact, $M /(H \cap M)$, being homeomorphic to $\pi(M)$, is compact. Then by [16, Theorem 6.2], $H \cap M$ is compactly generated. By assumption on $H, H \cap M$ is compact, hence consequently $M$ is compact. 
THEOREM 1.3. Let $G$ be a locally compact group and the groups $W_{\lambda}(G)$ defined as above. Then we have the following conditions:

(i) Every relatively compact subset $X$ of $W_{\lambda}(G)$ generates a relatively compact subgroup of $G$; in particular $W_{\lambda}(G) \subset P(G)$.

(ii) $\pi_{\lambda}^{-1}\left(P\left(G / W_{\lambda}(G)\right)\right)=P(G)$ where $\pi_{\lambda}: G \rightarrow G / W_{\lambda}(G)$ is the projection map.

Proof. By Lemma 1.2, (i) implies (ii), hence it suffices to show (i). We prove the assertion by a transfinite induction argument.

Case 1. $\lambda=1$. This is just (ii) of Theorem 1.1 in [17].

Case 2. $\lambda>1$ and is not a limiting ordinal number. Let $\alpha=\lambda-1$. Let $X$ be a relatively compact subset of $W_{\lambda}(G)$. Then $\pi_{\lambda}(X)$ is a relatively compact subset of $W_{1}\left(G / W_{\alpha}(G)\right)$. By Case 1 , the group $\left\langle\pi_{\lambda}(X)\right\rangle$ is relatively compact in $G / W_{\alpha}(G)$. Hence by Lemma 1.2 , the group $\langle X\rangle$ is relatively compact in $G$.

Case 3. $\lambda$ is a limiting ordinal number. First we prove that $W_{\lambda}(G)$ has a compact open subgroup. Let $H$ be an open subgroup of $W_{\lambda}(G)$ which is a projective limit of Lie groups. Let us write $H_{\nu}$ for $H \cap W_{\nu}(G)$. Clearly the groups $H_{\nu}(\nu<\lambda)$ are closed normal subgroups of $H$ and the union $\bigcup_{\nu<\lambda} H_{\nu}$ is dense in $H$ for $W_{\lambda}(G)=\operatorname{Cl}\left(\bigcup_{\nu<\lambda} W_{\nu}(G)\right)$. Now let $K$ be a compact normal subgroup of $H$ such that the quotient group $H / K$ is a Lie group. By passage from $H$ to $H^{0} K / K$, we may assume that $H$ is an analytic group. Therefore by Lemma 1.4 coming next, $H^{0} \mathrm{~K}$ is compact. Let $M=H^{0} \mathrm{~K}$. Clearly $M$ is a compact open subgroup of $W_{\lambda}(G)$, and $W_{\lambda}(G)=\bigcup_{\nu<\lambda} W_{\nu}(G) M$. Let $X$ be a relatively compact subset of $W_{\lambda}(G)$. Then there are finitely many elements $x_{1}, \ldots, x_{l}$ in some $W_{\nu}(G)$ with $\nu<\lambda$ such that $X$ is contained in the union $\bigcup_{i=1}^{l} x_{i} M$. Let $Y$ be the union of $C\left(M, x_{i}\right)(i=1, \ldots, l)$ where $C\left(M, x_{i}\right)=\left\{m x_{i} m^{-1}: m \in M\right\}$. Clearly $Y$ is relatively compact in $W_{\nu}(G)$, hence the group $\langle Y\rangle$ is relatively compact in $G$. Certainly $M$ normalizes $Y$ hence $\langle Y\rangle$. This shows that $M\langle Y\rangle$ is a relatively compact subgroup of $G$ containing $X$. Therefore the proof is completed.

LEMMA 1.4. Let $H$ be an analytic group and $H_{\nu}$ closed normal subgroups of $H$ such that $H_{\nu} \subset P(H)$ for $\nu<\lambda$ and $\bigcup_{\nu<\lambda} H_{\nu}$ dense in $H$. Then $H$ is compact.

Proof. $H_{\nu}^{0}$ contains only periodic elements. Then by [17, Theorem 5.2] and [16, Theorem 7.1],$H_{\nu}^{0}$ is compact. Since $H_{\nu} / H_{\nu}^{0}$ is discrete and central in $G / H_{\nu}^{0}$ and $H_{\nu} / H_{\nu}^{0} \subset P\left(G / H_{\nu}^{0}\right), H_{\nu} / H_{\nu}^{0}$ has to be a finite central subgroup of $G / H_{\nu}^{0}$. This implies that $H_{\nu}$ is compact. Let $K$ be a maximal compact subgroup of $H$. Since $H_{\nu}$ is compact normal in $H, H_{\nu} \subset K$ for all $\nu<\lambda$. As $\bigcup_{\nu<\lambda} H_{\nu}$ is dense in $H, H=K$ follows immediately.

As an immediate consequence of Theorem 1.3, we have the following theorem of the radical $W(G)$ of $P(G)$. 
THEOREM 1.5. Let $G$ be a locally compact group and $W(G)$ the radical of $P(G)$. Then we have the following conditions:

(i) $W(G)$ is a closed characteristic subgroup of $G$.

(ii) Every relatively compact subset $X$ of $W(G)$ generates a relatively compact subgroup $\langle X\rangle$ of $G$, in particular $W(G) \subset P(G)$.

(iii) $\pi^{-1}(P(G / W(G)))=P(G)$ where $\pi: G \rightarrow G / W(G)$ is the projection map.

(iv) $G / W(G)$ has no nontrivial compact normal subgroups; moreover $W(G)$ is the smallest closed normal subgroup with condition (iv).

(v) If $G$ has a maximal compact normal subgroup $K$, then $W(G)=K$.

Proof. (i) to (iii) are immediate from Theorem 1.3, since $W(G)=W_{\beta}(G)$ for some ordinal number $\beta$ with $W_{\beta}(G)=W_{\beta+1}(G)$.

(iv) By $\left[16\right.$, Corollary 5.6], $W_{1}(G)=\mathrm{Cl}(F(G) \cap P(G))$ is the closed subgroup of $G$ generated by all compact normal subgroups of $G$. Hence the first part of (iv) follows for $W(G)=W_{\beta}(G)$ with $W_{1}\left(G / W_{\beta}(G)\right)=\{e\}$. Now let $N$ be a closed normal subgroup of $G$ such that $G / N$ has no nontrivial compact normal subgroups. By [16, Corollary 5.6] and an easy induction argument, one shows readily that $W_{\nu}(G) \subset N$ for all ordinal numbers $\nu \leqslant \beta$.

(v) If $G$ has a maximal compact normal subgroup $K$, then $K \subset W_{1}(G) \subset$ $W(G)$ and clearly $W_{1}(G / K)=\{e\}$, hence by (iv) $W(G) \subset K$. Therefore $W(G)=K$ follows easily.

REMARK. The radical $W(G)$ of $P(G)$ is rather useful in the sense that the passage from $G$ to $G / W(G)$ kills more or less only compact normal subgroups of G. By (iii) of Theorem 1.5 , in $G / W(G)$ we have not introduced new periodic elements. By set theory, we know that $W_{\beta}(G)=W_{\beta+1}(G)$ holds for some ordinal number $\beta$. It may be interesting to find the smallest $\beta$ with $W(G)=W_{\beta}(G)$. In some cases, $W(G)=W_{1}(G)$ is possible. By (iv) of Theorem 1.5, W(G) can be defined as the intersection of all closed normal subgroups $N$ of $G$ such that $G / N$ has no nontrivial compact normal subgroups.

THEOREM 1.6. Let $G$ be a finitely generated discrete group. Then $W(G)=$ $W_{1}(G)$.

We shall postpone the proof until $\S 2$. Actually in $\S 2$, we are going to present a more general theorem of this type.

2. Closed subgroups of $G$ contained in $F(G)$. Let $G$ be a locally compact group and $F(G)$ the group of all conjugate-bounded elements of $G$. In this section, we are going to study closed subgroups of $G$ contained in $F(G)$.

LEMmA 2.1. Let $K$ be a nonempty compact subset of $F(G)$. Then there are 
a point $k$ in $K$ and a neighborhood $N$ of $k$ in $K$ such that the union $\mathrm{gNg}^{-1}(g \in G)$ is relatively compact in $G$.

Proof. Suppose that the assertion is false. Let $V$ be a symmetric open relatively compact neighborhood of $e$ in $G$. We are going to construct inductively two sequences $\left\{k_{n}\right\}$ and $\left\{g_{n}\right\}$ of elements in $K$ and in $G$ respectively such that the following conditions hold:

(i) $g_{n} k_{n} g_{n}^{-1} V(n=1,2, \ldots)$ are disjoint,

(ii) $g_{n} k_{m} g_{n}^{-1} \in g_{n} k_{n} g_{n}^{-1} V$ for all $m>n$.

Assume that we have elements $g_{i} \in G, k_{i} \in K(i=1, \ldots, n)$ satisfying the conditions

(i) $g_{i} k_{i} g_{i}^{-1} V(i=1, \ldots, n)$ are disjoint,

(ii) $g_{j} k_{i} g_{j}^{-1} \in g_{j} k_{j} g_{j}^{-1} V$ for $n \geqslant i>j \geqslant 1$.

Clearly there is a neighborhood $W$ of $k_{n}$ in $G$ such that $g_{i} g g_{i}^{-1} \in g_{i} k_{i} g_{i}^{-1} V$, for $g \in W, 1 \leqslant i \leqslant n$. Let $M=W \cap K$. The union of $g g^{-1}(g \in G)$ is not relatively compact in $G$ by assumption. Hence there is $k_{n+1}$ in $M$ and $g_{n+1}$ in $G$ such that

$$
g_{n+1} k_{n+1} g_{n+1}^{-1} \notin \bigcup_{i=1}^{n} g_{i} k_{i} g_{i}^{-1} V^{2}
$$

Clearly the elements $g_{i}, k_{i}(i=1, \ldots, n+1)$ satisfy the conditions of $\left(\Delta_{n+1}\right)$. Hence by induction, the existence of the sequences $\left\{k_{n}\right\}$ and $\left\{g_{n}\right\}$ with conditions of $(\Delta)$ follows. However $K$ is compact. The sequence $\left\{k_{n}\right\}$ has a limiting point $k$ in $K$. From condition (ii) of $(\Delta), g_{n} k g_{n}^{-1} \in g_{n} k_{n} g_{n}^{-1} \bar{V}$ for every $n$. Since $k \in F(G)$, the conjugacy class $C(G, k)$ containing $k$ is relatively compact. Hence there is a compact subset $L$ of $G$ containing $g k^{-1}(g \in G)$. Hence $g_{n} k_{n} g_{n}^{-1} \in$ $L \bar{V}$ for every $n$. But $L \bar{V}$ is compact, the sequence $\left\{g_{n} k_{n} g_{n}^{-1}\right\}$ has a limiting point $x$. It follows that $x$ lies in $g_{n} k_{n} g_{n}^{-1} V$ for infinitely many $n$. Certainly this contradicts condition (i) of $(\Delta)$. Therefore the assertion of the lemma has to be true.

The argument used in Lemma 2.1 is a modification of [21, Theorem 1]. In order to apply Lemma 2.1 to study closed subgroups of $G$ contained in $F(G)$ we need to know the local structure of a locally compact group.

Lemma 2.2. Let $G$ be a locally compact group. Then $G$ has a compact neighborhood $N$ of $e$ with the condition: If $W$ is a nonempty open subset of $N$, then the interior $\stackrel{\circ}{W}$ of $W$ in $G$ is nonempty. 
Proof. Locally $G$ has the structure $K \times L$ where $K$ is a compact group and $L$ a local Lie group. Now the assertion of the lemma is immediate.

THEOREM 2.3. Let $G$ be a locally compact group and $H$ a subgroup of $F(G)$. Then the following conditions are equivalent:

(i) $\mathrm{Cl}(H) \subset F(G)$.

(ii) For every relatively compact subset $X$ of $\mathrm{Cl}(H)$, the union of $\mathrm{gXg}^{-1}$ $(g \in G)$ is relatively compact in $G$.

(iii) For every subset $X$ of $H$ relatively compact in $G$, the union of $\mathrm{gXg}^{-1}$ $(g \in G)$ is relatively compact in $G$.

Proof. (i) $\Rightarrow$ (ii). Let $L=\mathrm{Cl}(H) \subset F(G)$. Let $V$ be a compact neighborhood of $e$ in $L$ with the condition in Lemma 2.2. By Lemma 2.1, there is a nonempty open subset $N$ of $V$ such that the union $\mathrm{gNg}^{-1}(g \in G)$ is relatively compact in $G$. By our choice of $V$, the interior $\stackrel{\circ}{N}$ of $N$ in $L$ is not empty. Let $M=$ $n^{-1} \stackrel{\circ}{N}$ for an $n \in \stackrel{\circ}{N}$. Obviously $M$ is an open neighborhood of $e$ in $L$. Since $n \in F(G)$, the union of $g \mathrm{Mg}^{-1}(g \in M)$ is again relatively compact. This shows that $L$ has a compact neighborhood $K$ of $e$ in $L$ such that $K$ is normal in $G$. Now let $X$ be a relatively compact subset of $L$. Clearly there are finitely many elements $x_{1}, \ldots, x_{l}$ in $L$ with $X \subset \bigcup_{i=1}^{l} x_{i} K$. Hence $\bigcup_{g \in G} g X g^{-1}$, being contained in the union of $C\left(G, x_{i}\right) K(i=1, \ldots, l)$, is relatively compact.

(ii) $\Rightarrow$ (iii) is immediate.

(iii) $\Rightarrow$ (i). Let $L=\mathrm{Cl}(H)$ and $V$ a relatively compact open neighborhood of $e$ in $L$. Let $T=H \cap V$. Clearly $T$ is dense in $V$ and $T$ is a subset of $H$ relatively compact in $G$. Hence $\bigcup_{g \in G} g T^{-1}$ is relatively compact, and consequently $V \subset \mathrm{Cl}(T) \subset \mathrm{Cl}\left(\bigcup_{g \in G} g T g^{-1}\right) \subset F(G)$. Thus $\mathrm{Cl}(H)=L \subset F(G)$.

In [16] the author proved that $F(G) \cap P(G)$ is a characteristic subgroup of $G$. However, in general, it is not closed in $G$. With the aid of Theorem 2.3, we can give the following criterion when $F(G) \cap P(G)$ is closed.

THEOREM 2.4. Let $G$ be a locally compact group. Then the following conditions are equivalent:

(i) $F(G) \cap P(G)$ is closed in $G$.

(ii) Every relatively compact subset $X$ of $\operatorname{Cl}(F(G) \cap P(G)$ ) is contained in a compact normal subgroup of $G$.

(iii) Every subset $X$ of $F(G) \cap P(G)$ relatively compact in $G$ is contained in a compact normal subgroup of $G$.

Proof. (i) $\Rightarrow$ (ii). Let $L=F(G) \cap P(G)$ and $L=\mathrm{Cl}(L)$. By [17, Theorem 1.1], $L$ has a compact open subgroup $K$. By Theorem 2.3, the union of $Y$ of 
$g K g^{-1}(g \in G)$ is relatively compact. Certainly $Y$ is contained in $L$, and by [17, Theorem 1.1] $Y$ is contained in a compact normal subgroup $N$ of $G$. Let $X$ be a relatively compact subset of $L$. Then $X$ is contained in the union of $x_{i} N$ for finitely many $x_{i} \in L(i=1, \ldots, l)$. Clearly the set $\bigcup_{i=1}^{l} C\left(G, x_{i}\right) N$ is a relatively compact subset of $L$. Again by [17, Theorem 1.1], it is contained in a compact normal subgroup of $G$.

(ii) $\Rightarrow$ (iii) is trivial.

(iii) $\Rightarrow$ (i). Let $L=\mathrm{Cl}(F(G) \cap P(G))$. By [17, Theorem 1.1], $L$ has a compact open subgroup $K$. Clearly the set $X=K \cap(F(G \cap P(G)))$ is dense in $K$ and $X$ is relatively compact in $G$. By (iii), $X$ is contained in a compact normal subgroup $N$ of $G$. Hence $K \subset N \subset F(G) \cap P(G)$ and consequently $F(G) \cap P(G)$ is closed.

Definition 2.5. Let $G$ be a locally compact group. We define $F_{0}(G)=$ $\{g \in G$ : the conjugacy class $C(G, g)$ is compact $\} . F_{0}(G)$ is just a characteristic subset of $G$.

THEOREM 2.6. Let $G$ be a compactly generated locally compact group and $L=F(G) \cap P(G)$ such that $L$ is closed in $G$. Then $\pi^{-1}\left(F_{0}(G / L)\right)$ is contained in $F(G)$, where $\pi: G \rightarrow G / L$ is the projection map.

Proof. Let $x \in \pi^{-1}\left(F_{0}(G / L)\right)$ and $H=\left\{g \in G: g x g^{-1} \in x L\right\}$. Since $\pi(x) \in F_{0}(G / L), G / H$, being homeomorphic to the conjugacy class $C(G / L, \pi(x))$ containing $\pi(x)$, is compact. By [16, Theorem 6.2], $H$ is compactly generated. Let $K$ be a compact generating subset of $H$, and $X=\left\{h x h^{-1} x^{-1}: h \in K\right\}$. Clearly $X \subset L$ and is relatively compact. Hence by Theorem $2.4, X$ is contained in a compact normal subgroup $N$ of $G$. Hence $h x h^{-1} \in x N, h \in K$. Since $N$ is a normal subgroup of $G$, it implies readily $h x h^{-1} \in x N$, for $h \in K^{l}$ where $l$ is any integer. Hence $C(H, x) \subset x N$. Since $G / H$ is compact, there is a compact subset $M$ of $G$ with $G=M H$. Therefore $C(G, x) \subset C(M, x) N$. As the latter is easily seen to be relatively compact, $x \in F(G)$ follows.

Theorem 2.6 is a generalization of Theorem 1.6. For in the case of discrete groups $F_{0}(G)=F(G)$ and $F(G) \cap P(G)$ is always closed in $G$. Now we present the proof of Theorem 1.6.

THEOREM 1.6. Let $G$ be a finitely generated discrete group. Then $W(G)=$ $F(G) \cap P(G)$, where $W(G)$ is the radical of $P(G)$ defined in $\S 1$.

Proof. Let $L=F(G) \cap P(G)$. By Theorem 2.6, $\pi^{-1}(F(G / L))=F(G)$ and by (ii) of Theorem 1.3, $P(G)=\pi^{-1}(P(G / L))$ where $\pi: G \rightarrow G / L$ is the projection map. Hence $F(G / L) \cap P(G / L)=\{e\}$. By definition of $W(G), W(G)=L$. 
3. Closed normal subgroups of $G$ generated by subgroups of $F(G)$. In this section, we are going to characterize the elements in $F(G)$. For this purpose, we study closed normal subgroups of $G$ generated by subgroups of $F(G)$. Some of the following lemmas are known. For completeness, we offer proofs here.

Lemma 3.1. Let $G$ be a locally compact group with $G=\operatorname{Cl}(F(G))$. Then we have the following conditions:

(i) The identity component $G^{0}$ of $G$ is contained in $F(G)$.

(ii) $G^{0}$ has a unique maximal compact subgroup $K$ such that $G^{0} / K$ is a central vector subgroup of $G / K$.

Proof. Let $H$ be an open subgroup of $G$ such that $H$ is a projective limit of Lie groups. Let $N$ be a compact normal subgroup of $H$ such that $H / N$ is a Lie group. Obviously then $F(H / N)$ is dense in $H / N$. Then by [16, Proposition 4.4], the lemma is true for $H / N$. Since $(H / N)^{0}=H^{0} N / N$, it follows that $G^{0}=H^{0}$ has a maximal compact normal subgroup $K$ such that $G^{0} / K$ is a vector subgroup of $G / K$. Now it is clear that in order to prove (i) and (ii) it suffices to show that $G^{0} / K$ is central $G / K$. Let $\alpha \in F(G / K)$ and $C_{\alpha}: G^{0} / K \rightarrow G^{0} / K$ the map defined by $C_{\alpha}(x)=\alpha x \alpha^{-1} x^{-1}\left(x \in G^{0} / K\right)$. Certainly $C_{\alpha}$ is a continuous endomorphism and the image, $\operatorname{Im}\left(C_{\alpha}\right)$, of $C_{\alpha}$ is relatively compact. Since $G^{0} / K$ is a vector group, $C_{\alpha}$ has to be trivial. As $F(G / K)$ is dense in $G / K, G^{0} / K$ is central in $G / K$.

LEMMA 3.2. Let $G$ be a locally compact group, $M$ a closed normal subgroup of $G$ with $M=\mathrm{Cl}(M \cap F(G))$ and $L=\mathrm{Cl}(M \cap F(G) \cap P(G))$. Assume that $M^{0}$ is a vector subgroup of $G$. Then $M^{0} \times L$ is an open subgroup of $M$.

Proof. Clearly $F(M)$, containing $M \cap F(G)$, is dense in $M$. Hence by Lemma $3.1, M^{0}$ is a central vector subgroup $V$ of $M$. Certainly $V \cap L$ is a closed subgroup of $V$, hence compactly generated. On the other hand by [17, Theorem 1.1], $V \cap L$ is compact and consequently $V \cap L=\{e\}$. Since $V$ is central in $M$ and $M / V$ is totally disconnected, $M$ has an open subgroup $H=V \times A$ where $A$ is a compact subgroup of $M$. Now let $x \in F(G) \cap H$ and $a(x)$ the projection of $x$ in $A$. Since $F(G) \cap H$ is dense in $H, A_{1}=\{a(x): x \in F(G) \cap H\}$ is dense in $A$. If $A_{1} \subset L$, then $A=\mathrm{Cl}\left(A_{1}\right) \subset L$ and consequently $V \times L$, containing $H$ is open in $M$. Hence it suffices to prove that $a(x) \in L$ for $x \in F(G) \cap H$. Let $\bar{x}$ be the image of $x$ in $G / V$. Clearly $\bar{x} \in F(G / V) \cap P(G / V)$. By [16, Theorem 3.5], $\bar{x}$ is contained in a compact normal subgroup of $G / V$. Let $N(x)$ be the smallest closed normal subgroup of $G$ containing $x$ and $V$. Clearly $N(x) \subset M$ and $N(x) / V$ is compact. As $V$ is a central vector group in $N(x)$ and $N(x) / V$ is compact, the extension splits, hence $N(x)=V \times B$ where $B$ is a compact subgroup of $M . B$, being characteristic in $N(x)$, is normal in $G$. In particular $A B$ is compact and 
$V \cap A B=\{e\}$. Let $b(x)$ (resp. $c(x))$ be the projection of $x$ in $B$ (resp. in $A B)$. Obviously $a(x)=c(x)=b(x) \in B \subset L$.

Lemma 3.3. Let $G, M$ and $L$ be as in Lemma 3.2. Then $M^{0} \times L \cap F(G)=$ $\left(M^{0} \cap F(G)\right) \times(L \cap F(G))$ and $M^{0} \subset F(G)$.

Proof. Let $M^{0}=V$. Since $V$ and $L$ are normal in $G$, and $V \times L$ is closed in $G$, it follows readily that $V \times L \cap F(G)=(V \cap F(G)) \times(L \cap F(G))$, hence $V \cap F(G)$ is dense in $V$. But $V$ is a vector group. By [16, Lemma 3.3], $V \subset F(G)$.

Lemma 3.4. Let $G, M$ and $L$ be as in Lemma 3.2. Then $M / M^{0} L$ is $a$ discrete torsion free abelian group.

Proof. Let $M^{0}=V$. By Lemma 3.2, VL is open in $M$, hence $M=$ $(F(G) \cap M) V L$. Suppose that $g \in F(G) \cap M$ with $g^{n} \in V L$ for some positive integer $n$. Let us write $g^{n} \in v l$, with $v \in V$ and $l \in L$. By Lemma 3.3, $v, l \in F(G)$. Let $w$ be the unique $n$th root of $v^{-1}$ in $V$. It is easy to see that $w \in F(G)$ and consequently $g w \in F(G)$. As $V$ is central in $M,(g w)^{n}=l \in L \subset P(G)$. Hence $g w$ lies in $M \cap F(G) \cap P(G)$. This shows that $M / V L$ is torsion free. Now $M / V L$ is a discrete torsion free $F C$-group, hence is abelian.

Lemma 3.5. Let $G, M$ and $L$ be as in Lemma 3.2. Then $M / L$ is the direct product of a vector group and a discrete torsion free abelian group.

Proof. Let $\alpha \in F(M / L)$. By Lemma 3.4, the map $c_{\alpha}$ defined by $c_{\alpha}(x)=$ $[\alpha, x]$ with $x \in M / L$ is a map from $M / L$ into $M^{0} L / L$. Let $V=M^{0}$. Since $V L / L$ is central in $M / L, c_{\alpha}$ is a continuous homomorphism and $\operatorname{Im}\left(c_{\alpha}\right)$ is relatively compact. Hence $c_{\alpha}$ is trivial, and as $F(M / L)$ is dense in $M / L, M / L$ is abelian. Now the conclusion follows from Lemma 3.4.

Lemma 3.6. Let $G, M$ and $L$ be as in Lemma 3.2. Then $M=V \times D$ where $V=M^{0}$ and $D$ a totally disconnected subgroup of $M$ with $D=\operatorname{Cl}(D \cap F(G))$.

Proof. By the preceding lemma, there is a closed subgroup $D$ of $M$ such that $M / L=V L / L \times D / L$. Clearly $M=V \times D$, and $D$ is totally disconnected for $M^{0}=V$. Since $F(G) \cap M$ is dense in $M$ and $V \subset F(G)$ by Lemma 3.3, hence $D \cap F(G)$ is dense in $D$.

LEMMA 3.7. Let $G$ be a compactly generated locally compact group such that $G=\mathrm{Cl}(F(G))$. Then $P(G)$ is a compact subgroup of $G$.

Proof. By Lemma 3.1, $G^{0}$ has a maximal compact normal subgroup $K$ such that $G^{0} / K$ is a central vector subgroup of $G / K$. By passage from $G$ to $G / K$, we may assume that $G^{0}$ is a vector group. By Lemma 3.6, $G=V \times D$ where $D$ 
is a totally disconnected subgroup of $G$ with $D=\operatorname{Cl}(D \cap F(G))$. Hence it suffices to show that $P(D)$ is a compact subgroup of $D$. Thus we may assume that $G$ is totally disconnected. Since $F(G)$ is dense in $G$, and $G$ is compactly generated, there are elements $g_{1}, \ldots, g_{l} \in F(G)$ and a compact open subgroup $K_{0}$ of $G$ such that $K_{0}, g_{1}, \ldots, g_{l}$ generate $G$. Now let

$$
X_{i}=\left\{\left[g_{i}, x\right]: x \in G\right\} \cup\left\{\left[g_{i}^{-1}, x\right]: x \in G\right\} .
$$

Clearly the sets $X_{i}$ are relatively compact in $G$ and, by Lemma 3.5, are contained in $L=\mathrm{Cl}(F(G) \cap P(G))$. Again by Lemma 3.5, $P(G / L)$ is trivial, hence $L=$ $P(G) \supset K_{0}$. Then by [17, Theorem 1.1], there is a compact subgroup $K$ of $G$ containing $K_{0}$ and the sets $X_{i}(i=1, \ldots, l)$. Since $K \supset X_{i}, K$ is normalized by $g_{i}$. Therefore $K$ is a compact open normal subgroup of $G$. Now $G / K$ is a finitely generated discrete $F C$-group. Hence $P(G / K)$ is a finite group and therefore $P(G)$ is the preimage of $P(G / K)$ in $G$ and consequently $P(G)$ is compact.

Summerizing the results, we can present them in

THEOREM 3.8. Let $G$ be a locally compact group, $M$ a closed normal subgroup of $G$ with $M=\mathrm{Cl}(M \cap F(G))$. Then we have the following conditions:

(i) $P(M)=\mathrm{Cl}(M \cap F(G) \cap P(G))$.

(ii) $M^{0} \subset F(G)$.

(iii) $M=(M \cap F(G)) P(M)$.

(iv) $M / P(M)$ is the direct product $V \times D$ where $V$ is a normal vector subgroup of $G / P(M)$ and $D$ a normal discrete torsion free abelian subgroup of $G / P(M)$ with $D \subset F(G / P(M))$.

(v) Let $K$ be the maximal compact normal subgroup of $M^{0} . M / K$ is the direct product of $W \times T$ where $W$ is a normal vector subgroup of $G / K$ and $T$ a totally disconnected normal subgroup of $G / K$ with $T=\mathrm{Cl}(T \cap F(G / K))$.

Proof. Clearly $F(M) \supset M \cap F(G)$ is dense in $M$. Hence by Lemma 3.1, $M^{0}$ has a maximal normal compact subgroup $K$ such that $M^{0} / K$ is a central vector subgroup of $M / K$. By passage from $G$ to $G / K$, we may assume that $K=\{e\}$ and $V=M^{0}$ is a vector subgroup of $G$. Let $L=\mathrm{Cl}(M \cap F(G) \cap P(G))$. Clearly $L \subset$ $\mathrm{Cl}(F(G) \cap P(G)) \subset P(G)$. However by Lemma 3.5, $P(M / L)=\{e\}$, hence $L=$ $P(M)$. Thus (i) is proved. (ii) follows just from Lemma 3.3. (iii) follows from (ii) and Lemma 3.6. Assertions (iv) and (v) except the part that $D$ (resp. $T$ ) is normal in $G / P(M)$ (resp. $G / K$ ) are Lemmas 3.5 and 3.6. Let $V=M^{0}$ and $L=$ $\mathrm{Cl}(M \cap F(G) \cap P(G))=P(M)$. Suppose that there is a closed normal subgroup $D$ of $G / L$ such that $M / L=V L / L \times D$. Then let $T$ be the preimage of $D$ in $G$. Clearly we have $M=V \times T$. Hence it suffices to show that there is a closed normal subgroup $D$ of $G / L$ with $M / L=V L / L \times D$. By passage from $G$ to $G / L$, 
we may assume further that $M$ is an abelian Lie group. Let $D$ be a maximal normal subgroup of $G$ contained in $M$ such that $V \cap D=\{e\}$. We claim that $M=V \times D$. Since $V$ is open in $M, D$ is discrete, hence closed in $M$. Consider then the groups $\bar{G}=G / D, \bar{M}=M / D$ and $\bar{V}=V D / D$. By (i) $P(\bar{M})$ is a characteristic subgroup of $M$ and clearly $V \cap P(M)=\{e\}$. Let $D_{1}$ be the preimage of $P(M)$ in $M$. Clearly $D_{1}$ is a normal subgroup of $G$ with $D_{1} \cap V=\{e\}$. By the maximality of $D$, we must have $D=D_{1}$, hence $P(\bar{M})=\{e\}$. Suppose that $\bar{V} \neq \bar{M}$. Since $F(\bar{G}) \cap \bar{M}$ is dense in $\bar{M},(\bar{M})^{0} \subset F(\bar{G})$ by (ii) and $(\bar{M})^{0}$ is open in $\bar{M}, \bar{M} \subset$ $F(\bar{G})$. Then by Theorem 2.3 , there is a compactly generated normal subgroup $N$ of $\bar{G}$ such that $\bar{V} \varsubsetneqq N \subset \bar{M}$. Since $N$ is a compactly generated abelian Lie group without periodic elements, $N$ can be identified with a closed subgroup of a vector group $A$ such that $N$ generates $A$ over R. Let $\operatorname{Aut}(N)$ (resp. GL $(A)$ ) be the automorphism group of $N$ (resp. of $A$ ). Clearly $\operatorname{Aut}(N)$ can be identified with the closed subgroup of $\mathrm{GL}(A)$ consisting of all $\alpha \in \mathrm{GL}(A)$ with $\alpha(N)=N$. Let $w$ : $\bar{G} \rightarrow \operatorname{Aut}(N)$ be the representation given by $w(g)(h)=g h g^{-1}(g \in \bar{G}, h \in N)$. Since $F(\bar{G}) \cap N$ is dense in $N, \mathrm{Cl}(w(\bar{G}))$ is compact in $\mathrm{GL}(A)$. It follows that $A=V \times B$ where $B$ is $w(\bar{G})$-invariant. Hence $N=\bar{V} \times(N \cap B)$. Obviously $N \cap B$ is normal in $\bar{G}$. Let $D^{\prime}$ be the preimage of $N \cap B$ in $G$. $D^{\prime} \supsetneqq D$ and $D \cap V=\{e\}$. This contradicts the maximality of $D$. Therefore $M=V \times D$ and the proof is completed.

COROLlARY 3.9. Let $G$ be a locally compact group and $N$ a closed compactly generated normal subgroup of $G$ with $N=\operatorname{Cl}(F(G) \cap N)$. Then we have the following conditions:

(i) $N$ has a unique maximal compact subgroup $K$.

(ii) $N / K$ is the direct product $V \times D$ where $V$ is a normal vector subgroup of $G / K$ and $D$ a discrete normal finitely generated torsion free abelian subgroup of $G / K$.

(iii) The index $\left[G / K: Z_{G / K}(D)\right]<\infty$ and $N / K \subset F(G / K)$.

(iv) $N \subset F(G)$.

Proof. (i) By Lemma 3.7, $P(N)$ is a compact subgroup of $N$, hence the maximal compact subgroup $K$ of $N$.

(ii) By (iv) of Theorem 3.8, (ii) is immediate.

(iii) Since $D$ is finitely generated, discrete, normal in $G / K$ and $D \subset F(G / K)$, $\left[G / K: Z_{G / K}(D)\right]<\infty$. Since $V \subset F(G / K)$ by (ii) of Theorem 3.8, $N / K \subset F(G / K)$ is immediate.

(iv) Since $K$ is compact normal in $G$ and $N / K \subset F(G / K)$, one concludes readily that $N \subset F(G)$. 
Applying the preceding corollary to elements in $F(G)$, we have the following criterion for elements in $F(G)$.

THEOREM 3.10. Let $G$ be a locally compact group. Then $g \in F(G)$ if and only if $g$ is contained in a closed normal subgroup $N$ of $G$ such that the following conditions hold:

(i) $N$ has a unique maximal compact subgroup $K$.

(ii) $N / K$ is the direct product $V \times D$ of a normal vector subgroup $V$ of $G / K$ and a normal discrete subgroup $D$ of $G / K$ isomorphic to $Z^{l}$ for some nonnegative integer $l$.

(iii) $\left[G / K: Z_{G / K}(D)\right]<\infty$ and $\alpha(G / K)$ is relatively compact in $\mathrm{GL}(V)$ where $\alpha: G / K \rightarrow \mathrm{GL}(V)$ is the adjoint representation.

REMARK. Groups with dense $F(G)$ have been studied in [21]. The following theorem is a generalization of the result of Tits [12] on $F(G)$.

THEOREM 3.11 [12]. Let $G$ be a locally compact group such that $G$ has no nontrivial compact normal subgroups. Then we have the following conditions:

(i) $F(G)$ is closed.

(ii) $F(G)$ is a direct product $V \times D$ of a normal vector subgroup $V$ of $G$ and a discrete torsion free abelian normal subgroup $D$ of $G$.

(iii) Let $\alpha \in A(G)$ such that $\left\{g^{-1} \alpha(g): g \in G\right\}$ is relatively compact. Then there are an element $x \in F(G)$ and an open normal subgroup $H$ of $G$ such that $[G: H]<\infty$ and $\alpha(h)=x h x^{-1}$ for all $h \in H$.

Proof. (i) and (ii) Let $M=\mathrm{Cl}(F(G))$ and $L=\mathrm{Cl}(F(G) \cap P(G))$. Since $G$ has no nontrivial compact normal subgroups, by [16, Corollary 5.6], $L=\{e\}$. Hence by Theorem 3.8, (i) and (ii) are immediate.

(iii) Let $A=\langle\alpha\rangle \cdot G$ be the semidirect product of an open normal subgroup $G$ and a discrete cyclic subgroup $\langle\alpha\rangle$ of $A$. From the condition on $\alpha, \alpha \in F(A)$. Hence by Theorem 3.10, there is a closed normal subgroup $N$ of $A$ containing $\alpha$ such that $N$ has a unique maximal compact subgroup $K$ and $N / K=V \times D$ is a direct product of a normal vector subgroup $V$ of $A / K$ and a normal finitely generated discrete torsion free abelian subgroup $D$ of $A / K$. Moreover we know that $\left[A / K: Z_{A / K}(D)\right]<\infty$. Let $H$ be the preimage of $Z_{A / K}(D)$ in $G$ and $x \in N^{0}$ such that the image $\bar{x}$ in $N / K$ equals the component $\bar{\alpha}_{V}$ of $\bar{\alpha}$ in $V$ where $\bar{\alpha}$ is the image of $\alpha$ in $N / K$. Clearly $x \in F(G)$ and $\left(x h x^{-1}\right) \alpha\left(h^{-1}\right) \in G \cap K=\{e\}$, $\forall h \in H$. Hence (iii) is proved.

4. Compactness and finiteness of Haar measure. In this section, we look at the compactness conditions related to finiteness conditions on Haar measure. 
Let $G$ be a locally compact group and $H$ a closed subgroup of $G$. $H$ is called uniform in $G$ if $G / H$ is compact. $H$ is said to have finite-invariant covolume in $G$ if $G / H$ has a finite invariant Haar measure. In general, these two conditions are not the same. However they are related. For example, a discrete uniform subgroup $H$ of $G$ always has a finite invariant covolume. In [18], the author proved that if $G$ is a Lie group such that the Lie algebra $\hat{G}$ of $G$ has no noncompact semisimple subalgebras, then a closed subgroup $H$ of $G$ with finite invariant covolume is always uniform in $G$. In this section, we are going to study groups with these two conditions being equivalent. First we recall some definitions and notations in measure theory. Let $G$ be a locally compact group and $A(G)$ the automorphism group of $G$ consisting of all bicontinuous automorphisms of $G$. Let $d g$ be a fixed left invariant Haar measure. For each $\alpha \in A(G)$, certainly $d \alpha(g)$ is also a left invariant Haar measure. Hence by the uniqueness theorem of Haar measure, there is a positive number $\Delta_{G}(\alpha)$ with $d \alpha(g)=\Delta_{G}(\alpha) d g$. Clearly the map $\alpha \rightarrow \Delta_{G}(\alpha)$ $(\alpha \in A(G))$ is a group homomorphism. For simplicity, we write $\Delta_{G}(x)=\Delta_{G}\left(w_{x}\right)$ with $x \in G$ where $w_{x}$ is the inner automorphism of $G$ given by $w_{x}: g \rightarrow x g x^{-1}$ $(g \in G)$. Let $H$ be a closed subgroup of $G$. It is well known that $G / H$ has an invariant measure if and only if $\Delta_{G}(h)=\Delta_{H}(h)$ for $h \in H$.

Proposition 4.1. Let $G$ be a locally compact group and $H$ a closed subgroup of $G$ with finite invariant covolume. If $\alpha \in A(G)$ with $\alpha(H)=H$, then $\Delta_{G}(\alpha)=\Delta_{H}(\bar{\alpha})$ where $\bar{\alpha}$ is the automorphism of $H$ induced by $\alpha$.

Proof. Let $\pi: G \rightarrow G / H$ be the projection map and $\bar{x}=\pi(x)$ for $x$ in $G$. Let $d h$ (resp. $d \bar{g}$ ) be a fixed left invariant Haar measure on $H$ (resp. on $G / H$ ). We know that $d g=d \bar{g} d h$ is a left invariant Haar measure on $G$. Let $f: G \rightarrow C$ be a continuous function with compact support and $\bar{f} G / H \rightarrow C$ given by

$$
\bar{f}(\bar{g})=\int_{H} f(g h) d h .
$$

Then we have the following equalities:

$$
\begin{aligned}
\Delta_{G}(\alpha) \int_{G} f(g) d g & =\int_{G} f\left(\alpha^{-1}(g)\right) d g \\
& =\int_{G / H} d \bar{g} \int_{H} f\left(\alpha^{-1}(g h)\right) d h=\Delta_{H}(\bar{\alpha}) \int_{G / H} \bar{f}\left(\pi\left(\alpha^{-1}(g)\right)\right) d \bar{g} .
\end{aligned}
$$

Hence $\Delta_{G}(\alpha) \int_{G / H} \bar{f}(\bar{g}) d \bar{g}=\Delta_{H}(\bar{\alpha}) \int_{G / H} \bar{f}\left(\pi\left(\alpha^{-1}(g)\right)\right) d \bar{g}$. It is true for all integrable functions $\bar{f}: G / H \rightarrow C$ by the limiting argument. Therefore $\Delta_{G}(\alpha) v(G / H)=$ $\Delta_{H}(\bar{\alpha}) v(G / H)$ if we take $\bar{f}$ to be the characteristic function on $G / H$. Since $v(G / H)$ is finite by assumption, $\Delta_{G}(\alpha)=\Delta_{H}(\bar{\alpha})$. 
Lemma 4.3. Let $G=H N$ be a locally compact group where $N$ is a closed normal subgroup of $G$ such that the canonical map $H / H \cap N \rightarrow G / N$ is a topological isomorphism. Let $\alpha: H \rightarrow A(H \cap N)$ (resp. $\beta: G \rightarrow A(N))$ be the representation defined by conjugation. Then for each $h \in H$,

$$
\Delta_{H}(h)=\Delta_{H / H \cap N}(\bar{h}) \Delta_{H \cap N}(\alpha(h)) \quad \text { and } \quad \Delta_{G}(h)=\Delta_{G / N}(\bar{h}) \Delta_{N}(\beta(h))
$$

where $\bar{h}$ is the image of $h$ in $H / H \cap N$ (resp. in $G / N)$; in particular $\Delta_{H}(h)=\Delta_{G}(h)$ if and only if $\Delta_{H \cap N}(\alpha(h))=\Delta_{N}(\beta(h))$.

Proof. Let $d n$ and $d \bar{g}$ be fixed Haar measures on $N$ and $G / N$ respectively. Then $d g=d \bar{g} d n$ is a Haar measure on $G$. It follows easily that $\Delta_{G}(h)=$ $\Delta_{G / N}(\bar{h}) \Delta_{N}(\beta(h))$. Similarly we have $\Delta_{H}(h)=\Delta_{H / H \cap N}(\bar{h}) \Delta_{H \cap N}(\alpha(h))$. Since $H / H \cap N \rightarrow G / N$ is an isomorphism, $\Delta_{G / N}(\bar{h})=\Delta_{H / N \cap N}(\bar{h})$, and the second assertion follows.

Proposition 4.4. Let $G=H N$ be a locally compact group where $N$ is a closed normal subgroup of $G$ and $H$ a closed subgroup of $G$ such that the canonical maps $H / H \cap N \rightarrow G / N$ and $N / N \cap H \rightarrow G / H$ are homeomorphisms. Then $H$ has a finite invariant covolume in $G$ if and only if $N \cap H$ has a finite invariant covolume in $N$.

Proof. $(\Rightarrow$ ) As the canonical map $N / N \cap H \rightarrow G / H$ is a homeomorphism commuting with translations by elements in $N$, hence the necessary condition is immediate.

$\Leftrightarrow$ By Lemma 4.1, $\Delta_{N \cap H}(\alpha(h))=\Delta_{N}(\beta(h))$ for $h \in H$. Hence by Lemma 4.3, $\Delta_{H}(h)=\Delta_{G}(h), h \in H$. This shows that $G / H$ has a invariant measure $v_{G / H}$. Since $N /(H \cap N) \rightarrow G / H$ is a homeomorphism commuting with translations by elements in $N$, hence $v_{G / H}$ induces an invariant measure $v$ on $N / H \cap N$. By assumption, $v(N /(H \cap N))<\infty$ and consequently $v_{G / H}(G / H)=v(N /(H \cap N))<\infty$.

THEOREM 4.5. Let $G$ be a Lie group and $H$ a closed subgroup of $G$ such that the Lie algebra $\hat{G}$ of $G$ has no noncompact semisimple subalgebras. Then the following two conditions are equivalent:

(i) $H$ is uniform in $G$.

(ii) $H$ has a finite invariant covolume in $G$.

Proof. (ii) $\Rightarrow$ (i) Certainly the index $\left[G: G^{0} H\right]<\infty$ and $G^{0} / G^{0} \cap H$ has a finite invariant measure. Hence by [18, Theorem 2.1], $G^{0} \cap H$ is uniform in $G^{0}$ and consequently $H$ is uniform in $G$.

(i) $\Rightarrow$ (ii) Clearly $G / H G^{0}$ has a finite invariant measure. Hence we may assume that $G=H G^{0}$, and by Proposition 4.4 we may assume that $G=G^{0}$. 
Let $R$ be the radical of $G . \mathrm{Cl}(H R) / R$ is a compact subgroup of the compact group $G / R$. Hence $G / \mathrm{Cl}(H R)$ has a finite invariant measure. Thus we may assume that $G=\mathrm{Cl}(H R)$. Let $H^{0}=K_{1} R_{1}$ be a Levi-decomposition of $H^{0}$ where $R_{1}$ is the radical of $H^{0}$ and $K_{1}$ a maximal compact semisimple analytic subgroup of $H^{0}$. Since $G=\mathrm{Cl}(H R), R_{1} \subset R$ and for any maximal compact analytic semisimple subgroup $K$ of $G$ containing $K_{1}, K_{1}$ is normal in $K$. Hence $K$ is an almost direct product $K=K_{1} K_{2}$ where $K_{2}$ is a normal analytic subgroup of $K$. Then we have $G=K_{1} K_{2} R$. Let $L=H \cap K_{2} R$. Clearly $L$ is uniform in $K_{2} R$. By Proposition 4.4, we can assume that $G=K_{2} R$ and $K_{1}=\{e\}$. Hence $H^{0}$ is solvable and consequently by a theorem of $\mathrm{L}$. Auslander, $\left({ }^{2}\right) G=(\mathrm{Cl} H R)^{0}$ is solvable. In the following $G$ is solvable. Let $M$ be the maximal normal analytic subgroup of $G$ contained in $H$. Clearly $M$ is closed in $G$. Consider then the group $G / M$. We can further assume that $M=\{e\}$. Then by a theorem of Mostow [8], $H \cap N$ is uniform in $N$ where $N$ is the nilradical of $G$. Since nilpotent Lie groups are unimodular, $N / H \cap N$ has an invariant Haar measure which is certainly finite, for $N / H \cap N$ is compact. By Proposition 4.4, $H N / H$ has a finite invariant measure. Since $G / H N$ is a compact albelian group, it has a finite invariant measure. Therefore $H$ has a finite invariant covolume in $G$.

5. Elements with compact conjugacy class. Let $G$ be a locally compact group and $F_{0}(G)$ the set of all $g$ in $G$ such that the conjugacy class containing $g$ is compact. It would be interesting to know when an element $g \in F(G)$ is in $F_{0}(G)$. In [18], it was proved that if $G$ is a Lie group and $\Lambda$ a subset of $A(G)$ such that the group $Z_{G}(\Lambda)$, defined by $\{g \in G: \lambda(g)=g$ for all $\lambda \in \Lambda\}$, has a finite invariant covolume in $G$, then $Z_{G}(\Lambda)$ is uniform in $G$. In this section, we are going to generalize this theorem to locally compact groups and ask its inverse.

LeMmA 5.1. Let $G$ be a connected locally compact group and $A$ a compact normal solvable subgroup of $G$. Then $A$ is central in $G$.

Proof. Case 1. $G$ is a compact analytic group. Then $G=T \cdot S$ where $T$ is a central torus and $S$ a compact semisimple analytic subgroup of $G$. Let $B=$ $S \cap T A$. Clearly $B$ is a normal solvable subgroup of $S$, hence central in $S$ and central in $G$. Now as $A \subset T B, A$ is central in $G$.

Case 2. $G$ is an analytic group. $A$ is contained in a maximal compact subgroup $K$ of $G . K$ is connected. Hence $A$ is abeiian by Case 1. Now $A$ is compact normal and abelian $G$. As $G$ is connected, $A$ is central in $G$.

Case 3. The assertion for the general case follows from the fact that $G$ is a projective limit of analytic groups.

$\left({ }^{2}\right)$ L. Auslander, Bieberbach's theorem on space groups, Amer. J. Math. 83 (1961), 279-280. 
Proposition 5.2. Let $G$ be a connected locally compact group and $K$ the maximal compact normal subgroup. Then there is a closed connected characteristic subgroup $G_{1}$ of $G$ such that $G=K G_{1}$ and $K \cap G_{1} \subset Z(G)$.

Proof. Case 1. $K \cap Z(G)=\{e\}$. There is an analytic subgroup $H$ of $Z_{G}(K)$ such that $G=K H$. Let $\mathscr{U}$ be the Lie algebra of $K \cap H$. Clearly $\mathscr{U}$ is an ideal of $\hat{H}$ and is reductive. Hence $\mathscr{U}$ is a direct product of $\mathscr{A}_{1} \oplus \mathscr{A}_{2}$ of an abelian ideal $\mathfrak{A}_{1}$ and a compact semisimple ideal $\mathfrak{A}_{2}$. It follows that $\hat{H}=\mathfrak{A}_{2} \oplus B$ for some ideal $B$ of $\hat{H}$, for $\mathfrak{A}_{2}$ is a semisimple ideal of $\hat{H}$. Let $G_{1}$ be the analytic subgroup of $H$ with Lie algebra $B$. Obviously $G=K G_{1}$ and $K \cap G_{1}$ has Lie algebra contained in $\mathscr{A}_{1}$ which is abelian. Hence by Lemma $5.1,\left(K \cap G_{1}\right)^{0}$ is central in $G$, thus is $\{e\}$ for $K \cap Z(G)=\{e\}$. Therefore $K \cap G_{1}$ is normal and totally disconnected in $G$, hence central in $G$ and consequently is $\{e\}$. It follows that $G=K \times G_{1}$. Let $\alpha$ be a continuous automorphism of $G$. We claim that $\alpha\left(G_{1}\right)=G_{1}$. Let $M=G_{1} \alpha\left(G_{1}\right)$. Certainly it is easy to see that $M$ is a normal analytic subgroup of $G$ and $M=(M \cap K) \times G_{1} . M \cap K \subset Z_{G}\left(G_{1}\right)$ and $M \cap K \subset$ $G_{1} \alpha(G) \subset Z_{G}(K)$, hence $M \cap K \subseteq K \cap Z(G)=\{e\}$. Hence $G_{1}=\alpha\left(G_{1}\right)$.

By Lemma 5.1, the group $G /(K \cap Z(G))$ has the condition in Case 1.

Case 2. By pulling back the assertion on $G /(K \cap Z(G))$ to the group $G$, the lemma follows.

As an immediate consequence to general locally compact groups, we have

Lemma 5.3. Let $G$ be a locally compact group. Then $G$ has an open subgroup $H$ such that $H$ has a maximal compact normal subgroup $K$ with $H=K G_{1}$ and $K \cap G_{1} \subset Z\left(G_{1}\right)$ where $G_{1}$ is a closed connected characteristic subgroup of $G$.

Let $G$ be a locally compact group and $\Lambda$ a subset of the automorphism group $A(G)$ of $G$. Let $L$ be a subgroup of $G$. We denote by $Z_{L}(\Lambda)$ the set $\{l \in L$ : $\lambda(l)=l$ for all $\lambda \in \Lambda$.

LEMmA 5.4. Let $G$ be a locally compact group, and $H=K G_{1}$ an open subgroup of $G$ where $K$ is the maximal compact normal subgroup of $H$ and $G_{1} a$ closed connected characteristic subgroup of $G$ with $K \cap G_{1} \subset Z\left(G_{1}\right)$. Let $\Lambda \subset$ $A(G)$ and $A=Z_{H}(\Lambda) K \cap G_{1}$. Then we have the following conditions:

(i) $A=\left\{g \in G_{1}: \exists k \in K\right.$ such that $\left.\lambda(g)=g k \lambda\left(k^{-1}\right) \forall \lambda \in \Lambda\right\}$.

(ii) $g^{-1} \lambda(g) \in Z\left(G_{1}\right) \cap K$ for all $g \in A$.

Proof. (i) is immediate.

(ii) $Z\left(G_{1}\right) \cap K$ is the maximal compact subgroup of $Z\left(G_{1}\right)$. Since $G_{1}$ is characteristic, $Z\left(G_{1}\right) \cap K$ is also a characteristic subgroup of $G$. Clearly by Lemma 5.1, $Z\left(G_{1} / Z\left(G_{1}\right) \cap K\right)$ has no periodic elements. Hence by passage from 
$G$ to $G /\left(Z\left(G_{1}\right) \cap K\right)$, we may assume that $K \cap Z\left(G_{1}\right)=\{e\}$. It follows that $H=$ $K \times G_{1}$, hence $K \subset Z_{G}\left(G_{1}\right)$ and consequently $\lambda(K) \subset Z_{G}\left(G_{1}\right)$. This shows that $g^{-1} \lambda(g) \in Z\left(G_{1}\right)$ for all $g \in A$. Thus the map $\bar{\lambda}: A \rightarrow Z\left(G_{1}\right)$ defined by $\bar{\lambda}(g)=$ $g^{-1} \lambda(g)(g \in A)$ is a continuous homomorphism. Since $\bar{\lambda}(A) \subset K \lambda(K) \cap Z\left(G_{1}\right)$. $\operatorname{Im}(\bar{\lambda})$ is relatively compact. However $Z\left(G_{1}\right)$ has no periodic elements, $\bar{\lambda}$ is trivial, hence $g^{-1} \lambda(g)=e$ for all $g \in A$.

Lemma 5.5. Let $G, H, K, G_{1}$ and $\Lambda$ be as in Lemma 5.4, and $B=$ $Z_{G_{1}}\left(A, Z\left(G_{1}\right)\right)$ given by $\left\{g \in G_{1}: g^{-1} \lambda(g) \in Z\left(G_{1}\right)\right.$ for all $\left.\lambda \in \Lambda\right\}$. Then $A$ is normal in $B$.

Proof. Let $g_{1} \in A$ and $g_{2} \in B$. Then there is $k \in K$ such that $\lambda\left(g_{1}\right)=$ $g_{1} k \lambda\left(k^{-1}\right)$ for all $\lambda \in \Lambda$. We see easily that

$$
\lambda\left(g_{2} g_{1} g_{2}^{-1}\right)=g_{2} \lambda\left(g_{1}\right) g_{2}^{-1}=g_{2} g_{1} g_{2}^{-1} k \lambda\left(k^{-1}\right) \text { for all } \lambda
$$

for $k \lambda\left(k^{-1}\right)=g_{1}^{-1} \lambda\left(g_{1}\right) \in Z\left(G_{1}\right)$. Hence by (i) of Lemma 5.4, $g_{2} g_{1} g_{2}^{-1} \in A$. This proves that $A$ is normal in $B$.

THEOREM 5.6. Let $G$ be a locally compact group and $\Lambda$ a subset of $A(G)$. If $Z_{G}(\Lambda)$ has a finite invariant covolume in $G$, then $G$ has an open subgroup $H$ such that the group $Z_{H}(\Lambda)$ is uniform in $H$.

Proof. Let $H$ be an open subgroup of $G$ such that $H$ has a maximal compact normal subgroup $K$ of $H$ and $H=K G_{1}$ for a closed connected characteristic subgroup $G_{1}$ of $G$ with $K \cap G_{1}=K \cap Z\left(G_{1}\right)$. Clearly $H / Z_{H}(\Lambda)$ has a finite invariant volume. $H=K G_{1}$, and $K$ is compact normal in $H$. It suffices to show that $H / Z_{H}(\Lambda) K$ is compact. Let $A=G_{1} \cap Z_{H}(\Lambda) K$. Clearly the canonical map $G_{1} / A \rightarrow H / Z_{H}(\Lambda) K$ is homeomorphism commuting with translations by elements in $G_{1}$. Since $H / Z_{H}(\Lambda) K$ has a finite invariant volume, so does $G_{1} / A$. Let $\bar{G}_{1}=$ $G_{1} / Z\left(G_{1}\right), \bar{\Lambda}=\left\{\bar{\lambda}: \bar{\lambda} \in A\left(G_{1}\right)\right.$ induced by $\left.\lambda, \lambda \in \Lambda\right\}$. Consider then the group $Z_{\bar{G}_{1}}(\bar{\Lambda})$. The preimage of $A_{\bar{G}_{1}}(\bar{\Lambda})$ in $G_{1}$ is just given by $B=Z_{G_{1}}\left(\Lambda, Z\left(G_{1}\right)\right)=$ $\left\{g \in G_{1}: g^{-1} \lambda(g) \in Z\left(G_{1}\right)\right.$ for all $\left.\lambda \in \Lambda\right\}$. Clearly $A \subset B$. Hence $\bar{G}_{1} / Z_{G_{1}}(\bar{\Lambda})$ has a finite invariant volume. Since $\bar{G}_{1}$ is a Lie group, by [18, Theorem 3.6] $Z_{\bar{G}_{1}}(\Lambda)$ is uniform in $\bar{G}_{1}$. Hence $G_{1} / B$ is compact. Now by Lemma $5.5, B / A$ is a locally compact group with a finite Haar measure. It is well known that $B / A$ is compact and consequently $G_{1} / A$ is compact. $H / Z_{H}(\Lambda) K$, being homeomorphic to $G_{1} / A$, is thus compact. Hence the proof is completed.

COROLlary 5.7. Let $G$ be a locally compact group, $\Lambda \subset A(G)$ such that the group $Z_{G}(\Lambda)$ has a finite invariant covolume in $G$. If $F\left(G / G^{0}\right)$ is open in $G / G^{0}$, then $Z_{G}(\Lambda)$ is uniform in $G$. 
Proof. Since $F\left(G / G^{0}\right)$ is open in $G / G^{0}, G / G^{0}$ has a compact open normal supbgroup $\bar{L}$. Let $L$ be the preimage of $\bar{L}$ in $G$. By Theorem $5.6, L / Z_{L}(\Lambda)$ is easily seen to be compact. Since $Z_{G}(\Lambda) L$ is an open subgroup of $G$, the index $\left[G: Z_{G}(\Lambda) L\right]$ is finite, hence $G / Z_{G}(\Lambda)$ is compact.

Now let $G$ be a locally compact group and $g \in F(G)$. By Theorem 3.10, there is a closed normal subgroup $N$ containing $g$ such that $N$ has a unique maximal compact subgroup $K$ and $N / K$ is the direct product $V \times D$ of a normal vector subgroup of $G / K$ and a normal discrete subgroup $D$ of $G / K$ isomorphic to $Z^{l}$ for some nonnegative integer $l$ with $N / K \subset F(G / K)$. Let $Z_{G}(N / K)$ (resp. $Z_{G}(g, K)$ ) be the group $\left\{x \in G: x n x^{-1} \in n K\right.$ for all $\left.n \in N\right\}$ (resp. $\left\{x \in G: x g x^{-1} \in g K\right\}$ ).

LemMA 5.8. Let $G$ be a locally compact group, $g \in F(G)$, and $K, N$ be described as above. Then we have the following conditions:

(i) $Z_{G}(g) \subset Z_{G}(g, K)$ and $Z_{G}(N, K) \subset Z_{G}(g, K)$.

(ii) The group $G / Z_{G}(N, K)$ is a Lie group with compact Lie algebra.

Proof. (i) is immediate.

(ii) There is a continuous injective homomorphism $\alpha: G / Z_{G}(N, K) \rightarrow$ $\operatorname{Aut}(N / K)$. Since $\operatorname{Aut}(N / K)$ is a Lie group, by [2, Proposition 1 in $\S \mathrm{XIV}]$, $G / Z_{G}(N, K)$ is a Lie group. Since $N / K \subset F(G / K), \operatorname{Im}(\alpha)$ is relatively compact in $\operatorname{Aut}(N / K)$. Hence the Lie algebra of $G / Z_{G}(N, K)$ is a compact Lie algebra.

THEOREM 5.9. Let $G$ be a locally compact group such that compact normal subgroups of $G$ are central and $g \in F(G)$. Then the following two conditions are equivalent:

(i) $Z_{G}(g)$ is uniform in $G$.

(ii) $Z_{G}(g)$ has a finite invariant covolume in $G$.

Proof. Let $K$ be a compact normal subgroup of $G$ with conditions in Lemma 5.8.

(i) $\Rightarrow$ (ii) $G / Z_{G}(N, K)$ is a Lie group with compact Lie algebra. Clearly $G / Z_{G}(g, K)$ is uniform. Hence by Theorem $4.5, G / Z_{G}(g, K)$ has a finite invariant measure. Now as $K$ is central in $G, Z_{G}(g)$ is normal in $Z_{G}(g, K)$. Hence $Z_{G}(g, K) / Z_{G}(g)$ is a compact group, thus has a finite invariant measure. As an immediate consequence, $G / Z_{G}(g)$ has a finite invariant measure.

(ii) $\Rightarrow$ (i) Reversing the argument in (i) $\Rightarrow$ (ii), we get readily that $Z_{G}(g)$ is uniform in $G$.

ADDED IN PROOF. The author would like to mention the interesting work by S. Grosser and M. Moskowitz, Compactness conditions in topological groups, J. Reine Angew. Math. 50 (1971), 1-40. Many results in $\$ 3$ were discussed in [21] (e.g. 3.1, 3.4, 3.7). 


\section{REFERENCES}

1. R. Baer, Finiteness properties of groups, Duke, Math. J. 15 (1948), 1021-1032. MR 10, 352 .

2. C. Chevalley, Theory of Lie groups. Vol. 1, Princeton Math. Ser., vol. 8, Princeton Univ. Press, Princeton, N. J., 1946. MR 7, 412

3. M. Goto, Dense imbeddings of locally compact groups, Ann. of Math. (2) 61 (1955), 154-169. MR 16, 447.

4. - A remark on a theorem of A. Weil, Proc. Amer. Math. Soc. 20 (1969), 163-165. MR 38 \#1211.

5. S. Grosser and Martin Moskowitz, On central topological groups, Bull. Amer. Math. Soc. 72 (1966), 826-830. MR 33 \#2764.

6. K. Iwasawa, On some types of topological groups, Ann. of Math. (2) 50 (1949), 507-558. MR 10, 679.

7. - Topological groups with invariant compact neighborhoods of the identity, Ann. of Math. (2) 54 (1951), 345-348. MR 13, 206, 1139.

8. G. D. Mostow, Homogeneous space with finite invariant measure, Ann. of Math. (2) 75 (1962), 17-37. MR 26 \#2546.

9. B. H. Neumann, Groups with finite classes of conjugate elements, Proc. London Math. Soc. (3) 1 (1951), 178-187. MR 13, 316.

10. L. S. Pontrjagin, Continuous groups, GITTL, Moscow, 1938; English transl., Topological groups, Princeton Math. Ser., vol. 2, Princeton Univ. Press, Princeton, N. J., 1939. MR 1, 44.

11. C. L. Siegel, Discontinuous groups, Ann. of Math. (2) 44 (1943), 674-689. MR 5, 228.

12. J. Tits, Automorphismes à déplacement borné des groupes de Lie, Topology 3 (1964), 97-107. MR 28 \#2170.

13. V. I. UŠakov, $A$ certain class of topological groups, Dokl. Akad. Nauk SSSR 144 (1962), 65-68 = Soviet Math. Dokl. 3 (1962), 682-685. MR 25 \#143.

14. — Topological $\bar{F} \bar{C}$-groups, Sibirsk. Mat. Ž. 4 (1963), 1162-1174. (Russian) MR $29 \# 2322$.

15. - Classes of conjugate subgroups in topological groups, Dokl. Akad. Nauk SSSR 190 (1970), 51-53 = Soviet Math. Dokl. 11 (1970), 48-50.

16. S. P. Wang, Compactness properties of topological groups, Trans. Amer. Math. Soc. 154 (1971), 301-314. MR 42 \#6152.

17. - Compactness properties of topological groups. II, Duke Math. J. 39 (1972), 243-251. MR 45 \#5270.

18. - Homogeneous spaces with finite invariant volume (to appear).

19. A. Weil, $L$ 'intégration dans les groupes topologigues, 2nd ed., Hermann, Paris, 1965.

20. T. S. Wu, A class of locally compact totally disconnected topological groups, Proc. Amer. Math. Soc. 23 (1969), 613-614.

21. T. S. Wu and Y. K. Yu, Compactness properties of topological groups, Michigan Math. J. 19 (1972), 299-313. MR 47 \#6938.

\section{DEPARTMENT OF MATHEMATICS, PURDUE UNIVERSITY, WEST LAFAYETTE, INDIANA 47907}

